\title{
PRODUCCIÓN CIENTIIFICA Y REDES DE COLABORACIÓN EN CÁNCER EN EL PERÚ 2000-2011: UN ESTUDIO BIBLIOMÉTRICO EN SCOPUS Y SCIENCE CITATION INDEX
}

\author{
Percy Mayta-Tristán ${ }^{1, a}$, Charles Huamaní2,b, Juan José Montenegro-Idrogo ${ }^{3, c}$, \\ César Samanez-Figari, ${ }^{4, d}$, Gregorio González-Alcaide ${ }^{5, \mathrm{e}}$
}

RESUMEN

Se realizó un estudio bibliométrico para describir la producción científica peruana en cáncer en revistas de visibilidad internacional, y evaluar las redes de colaboración científica. Se incluyó los artículos publicados sobre cáncer hechos en Perú en el periodo 2000 a 2011 en revistas indizadas en SCOPUS o Science Citation Index Expanded. Se identificaron 358 artículos, evidenciándose un incremento en la producción de cuatro artículos en el 2000 a 57 en el 2011. Los cánceres más estudiados fueron los de cuello uterino (77 publicaciones); mama (53), y estómago (37). El Instituto Nacional de Enfermedades Neoplásicas (INEN) fue la institución más productiva (121 artículos) y con mayor número de colaboraciones (180 instituciones distintas). Se identificaron 52 ensayos clínicos, 29 con al menos un autor del INEN. En conclusión, la investigación en cáncer en Perú se está incrementando, el INEN es la institución más productiva, con importante participación en ensayos clínicos.

Palabras clave: Neoplasias; Bibliometría; Investigación biomédica; Evaluación de la investigación en salud; Perú (fuente: DeCS BIREME).

\section{SCIENTIFIC PRODUCTION AND CANCER-RELATED COLLABORATION NETWORKS IN PERU 2000-2011: A BIBLIOMETRIC STUDY IN SCOPUS AND SCIENCE CITATION INDEX}

\begin{abstract}
A bibliometric study was carried out to describe the scientific production on cancer written by peruvians and published in international health journals, as well as to assess the scientific collaboration networks. It included articles on cancer written in Peru between the years 2000 and 2011 and published in health journals indexed in SCOPUS or Science Citation Index Expanded. In the 358 articles identified, an increase in the production was seen, from 4 articles in 2000 to 57 in 2011. The most studied types were cervical cancer (77 publications); breast cancer (53), and gastric cancer (37). The National Institute of Neoplastic Diseases (INEN) was the most productive institution (121 articles) and had the highest number of collaborations (180 different institutions). 52 clinical trials were identified, 29 of which had at least one author from INEN. We can conclude that, cancer research is increasing in Peru, the INEN being the most productive institution, with an important participation in clinical trials.
\end{abstract}

Key words: Neoplasms; Bibliometrics; Biomedical research; Health research evaluation; Peru (source: MeSH NLM).

\section{INTRODUCCIÓN}

El cáncer es un problema de salud pública mundial. Para el 2008 se estimó 12,7 millones de casos de cáncer y 7,6 millones de muertes por esta causa, de los cuales el $64 \%$ ocurrieron en países en desarrollo como el Perú (1). Ello ha motivado la expansión de los servicios de prevención, diagnóstico y tratamiento de los cánceres más frecuentes ${ }^{(2)}$ así como la realización de investigaciones que permitan encontrar mejores aproximaciones a esta problemática ${ }^{(3)}$.

La investigación requiere de políticas que ayuden a definir las líneas de investigación y que se favorezca la producción de conocimiento en las áreas que sean de interés para los estados ${ }^{(4)}$. Para lograrlo, es

\footnotetext{
Escuela de Medicina, Universidad Peruana de Ciencias Aplicadas. Lima, Perú.

Oficina General de Información y Sistemas, Instituto Nacional de Salud. Lima, Perú.

Facultad de Medicina Humana "San Fernando", Universidad Nacional Mayor de San Marcos. Lima, Perú.

Departamento de Investigación, Instituto Nacional de Enfermedades Neoplásicas. Lima, Perú.

Departamento de Historia de la Ciencia y Documentación, Universitat de València. Valencia, España.

Médico salubrista, ${ }^{\mathrm{b}}$ médico cirujano, ${ }^{\mathrm{c} i n t e r n o ~ d e ~ M e d i c i n a, ~}{ }^{\mathrm{d}}$ médico oncólogo, ${ }^{\mathrm{e}}$ historiador, doctor en documentación

Recibido: 19-02-13 Aprobado: 06-03-13
}

Citar como: Mayta-Tristán P, Huamaní C, Montenegro-Idrogo JJ, Samanez-Figari C, González-Alcaide G. Producción científica y redes de colaboración en cáncer en el Perú 2000-2011: un estudio bibliométrico en Scopus y Science Citation Index. Rev Peru Med Exp Salud Publica. 2013;30(1):31-6. 
fundamental la colaboración científica, que consiste en la interacción de investigadores para intercambiar habilidades, competencias o recursos, ello permite resultados superiores a los obtenidos mediante el trabajo individual ${ }^{(5)}$. Esta colaboración cobra especial importancia en países que no disponen de recursos para generar investigaciones que atiendan sus necesidades de investigación (6), particularmente en cáncer donde las investigaciones requieren del trabajo multidisciplinario. En este escenario, se constituyó la Red de Institutos Nacionales de Cáncer de la Unión de Naciones Suramericanas, que tiene como objetivo fomentar el intercambio de conocimiento y el desarrollo de programas de cooperación.

Es importante examinar cómo funcionan estas colaboraciones y su respectiva producción mediante el análisis de sus redes de colaboración y publicaciones científicas. Nuestro objetivo es describir la producción científica peruana en cáncer en revistas de alto impacto y evaluar las redes de colaboración.

\section{EL ESTUDIO}

Se realizó un estudio bibliométrico, que incluyó artículos publicados en revistas indizadas en SCOPUS o en Science Citation Index Expanded del ISI/Web of Knowledge (ISI) entre los años 2000 a 2011, que tuvieran como temática de interés el cáncer y con al menos un autor con filiación de país "Perú", o que el estudio haya sido realizado en el Perú o con muestras recolectadas en el Perú. Se definió como primer periodo 2000-2005, y como segundo periodo 2006-2011.

Se seleccionó la base de datos SCOPUS porque incluye todas las revistas MEDLINE, tiene mejor cobertura de revistas latinoamericanas (7) y registra la filiación institucional de todos los autores. Se complementó la búsqueda en ISI porque es más selectiva y es empleada en los rankings mundiales de producción científica ${ }^{(8)}$.

En ambas se usó la misma estrategia de búsqueda, que consistió en seleccionar aquellos artículos que tengan al menos una de las siguientes palabras clave: cáncer, tumor, neoplasia u oncología en el título, resumen o palabras clave, y que además tuvieran la palabra Perú o que uno de sus autores tenga como filiación institucional una institución peruana.

Las publicaciones obtenidas fueron exportadas como hoja de cálculo al programa Excell $\circledR$. Se procedió a eliminar las publicaciones duplicadas en SCOPUS e ISI. Se excluyó los resúmenes de congreso, libros o capítulos de libro, y artículos que no se referían a cáncer, o no estaban relacionados al Perú mediante la revisión de los resúmenes y, de ser necesario, el artículo a texto completo por dos autores independientemente.

Para cada artículo se registró el título y tópico de la revista, idioma y año de publicación, institución y país del autor corresponsal, tipo de publicación (original, reporte de caso, carta al editor, otros). En los artículos originales se evaluó si fue observacional o experimental, y dentro de estos, si era un ensayo clínico.

De acuerdo al resumen se construyó la variable "localización del cáncer", que fue agrupada teniendo en cuenta las tasas de mortalidad por cáncer en el Perú (9). Los artículos que se referían al cáncer en general, o incluían varias localizaciones a la vez, fueron asignados a la categoría "más de una localización". Se identificó las instituciones y países participantes de acuerdo a una metodología previamente descrita ${ }^{\left({ }^{(8)}\right.}$. Se recolectó la firma institucional (la institución a la que el autor declara estar afiliado, que podía ser más de una); firma país; autoría peruana (al menos uno de los autores tenía como firma país Perú); autoría colaborativa (participación de dos o más firmas institucionales en un artículo), y colaboración internacional (participación de dos o más firmas país en un artículo).

Se determinó las instituciones más productivas, el número de colaboraciones entre ellas (firmas que se relacionan entre sí por el número de publicaciones conjuntas); el número de publicaciones por año, y el porcentaje de artículos en los que el autor corresponsal con firma país "Perú". Los datos fueron procesados con el programa estadístico STATA v.11.0 para el análisis descriptivo. Se generó las redes de colaboración en el programa Pajek v.3.0.2, usando el algoritmo Kamada-Kawai, ponderando la intensidad de las colaboraciones (10). Para favorecer la visualización de las redes de colaboración, para las redes de instituciones, se grafican las instituciones con más de diez artículos y todas las colaboraciones.

\section{HALLAZGOS}

Se obtuvo 692 publicaciones con la búsqueda formulada. Luego de excluir artículos duplicados, que no trataban del tema o no relacionados a Perú, quedaron 358 publicaciones. El número de publicaciones se incrementó en forma sostenida de 4 en el año 2000 a 57 en el 2011, con una mediana de 25 artículos por año y un rango intercuartílico de 16,5 a 50,8 . El porcentaje de publicaciones que tenían como autor corresponsal a un investigador con filiación 


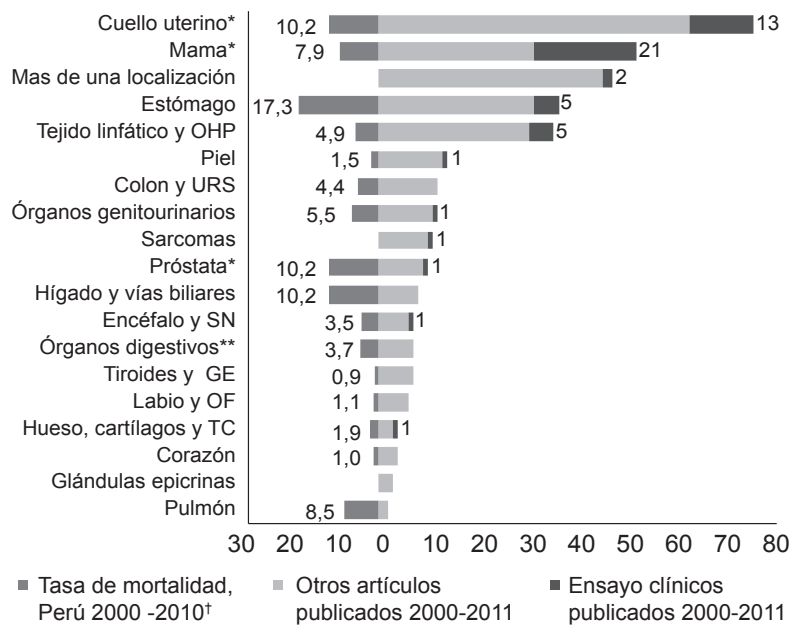

Figura 1. Tipo de artículo publicado según localización del cáncer y tasa de mortalidad.

OHP: órganos hematopoyéticos, URS: unión rectosigmoidea, SN: sistema nervioso, GE: glándulas endocrinas, OF: orofaringe, TC: tejido conjuntivo. *Tasas de mortalidad solo del sexo afectado: mujer para mama y cuello uterino, y varón para próstata.

Tasa de mortalidad por 100 mil habitantes, datos referenciales ${ }^{(9)}$.

** No incluye estómago

peruana fue de $29,9 \%$, siendo menor en 2000 con $0 \%$, y mayor en 2007 con $44,4 \%$.

El $82,1 \%$ de las publicaciones fueron en inglés, seguido del español (16,5\%), una en francés y cuatro en portugués.
Se publicó 256 (71,5\%) artículos de investigación; 38 $(10,6 \%)$ reportes de caso; $11(3,1 \%)$ cartas al editor, y 53 entre editoriales, artículos de revisión y otros. Las publicaciones que no eran artículos de investigación aumentaron de $20,6 \%$ en el primer periodo (2000-2005) a $31,4 \%$ en el segundo periodo (2006-2011).

Según la localización del cáncer se publicaron más artículos sobre cáncer de cuello uterino (77); mama (53); estómago (37) y tejido linfático, y órganos hematopoyéticos (36), así como aquellos que incluyeron más de una localización (48). Al compararlo con el reporte de tasa de mortalidad por cáncer hay localizaciones con alta mortalidad que tienen pocas publicaciones, como es el caso de pulmón, hígado y próstata (Figura 1).

Se identificó 194 revistas donde se realizó al menos una publicación. La mayoría $(66,2 \%)$ de artículos se publicaron en revistas en las que el cáncer no era el tema principal (Tabla 1). La revista donde se encontró más publicaciones fue la Revista de Gastroenterología del Perú (34), seguida del Journal of Clinical Oncology (14).

Se encontró 52 ensayos clínicos, los cuales fueron más frecuentes en el segundo periodo. En la mayoría de casos el autor corresponsal fue extranjero, fueron estudios colaborativos, particularmente con colaboración internacional, la mayoría realizados con coautoría de un

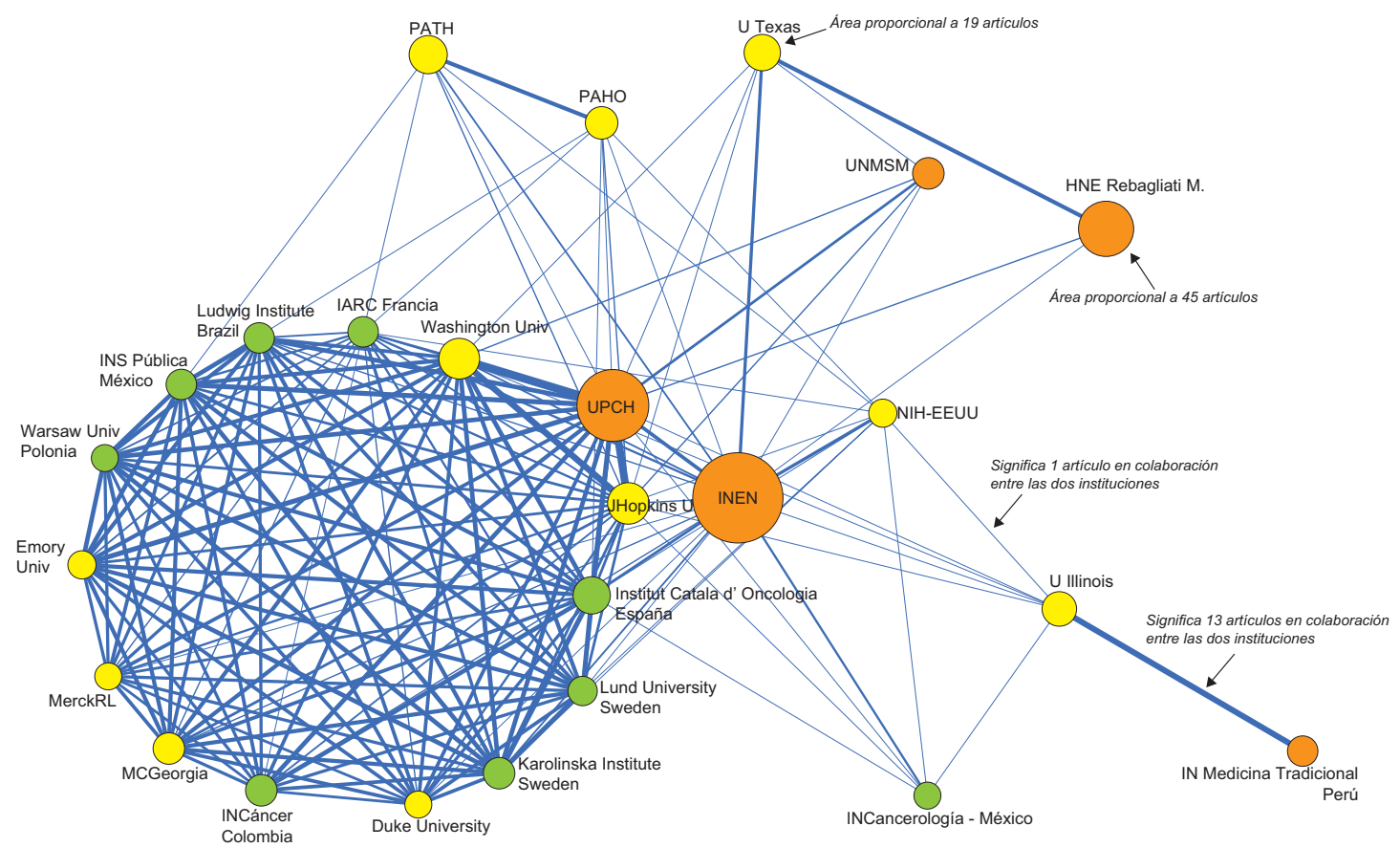

Figura 2. Instituciones participantes y sus vínculos en la red de colaboración peruana en investigación de cáncer.

Nota: las instituciones son representadas a través de círculos o "nodos", cuyos tamaños son proporcionales a los artículos que han producido, y la colaboración entre dos instituciones se representa como una línea que los une, cuyo grosor es proporcional a los trabajos en colaboración. Se muestran las instituciones con más de diez artículos y todas las colaboraciones entre ellos. 
Tabla 1. Revistas indizadas en SCOPUS o ISI que publican artículos peruanos sobre cáncer, 2000-2011.

\begin{tabular}{|c|c|c|c|}
\hline \multicolumn{2}{|l|}{ Revistas generales } & \multicolumn{2}{|l|}{ Revistas de cáncer } \\
\hline Título & N. ${ }^{\circ}$ artículos & Título & N. ${ }^{\circ}$ artículos \\
\hline Revista de Gastroenterología del Perú & 34 & Journal of Clinical Oncology & 14 \\
\hline Vaccine & 9 & International Journal of Cancer & 7 \\
\hline Journal of Natural Products & 8 & Annals of Oncology & 4 \\
\hline Actas Urológicas Españolas & 7 & Lancet Oncology & 4 \\
\hline Journal of Lower Genital Tract Disease & 6 & Leukemia and Lymphoma & 4 \\
\hline International Journal of Ginecology and Obstetrics & 5 & British Journal of Cancer & 3 \\
\hline Planta Medica & 5 & British Journal of Haematology & 3 \\
\hline Lancet & 4 & Cancer & 3 \\
\hline Progress in Biomedica & 4 & Clinical Cancer Research & 3 \\
\hline Revista Panamericana de Salud Pública & 4 & International Journal of Gynecological Cancer & 3 \\
\hline Revistas con tres artículos (4) & 12 & Tumori & 3 \\
\hline Revistas con dos artículos (23) & 46 & Revistas con dos artículos (17) & 34 \\
\hline Revistas con un artículo (93) & 93 & Revistas con un artículo (36) & 36 \\
\hline Total & 237 & Total & 121 \\
\hline
\end{tabular}

autor del INEN, y publicados en inglés y en revistas de cáncer (Tabla 2).

Se identificó la participación de 91 países, 26 de ellos solo colaboraron en un artículo, 26 colaboraron en más de 10 artículos y en 29 artículos no hubo un autor peruano. Los países con mayor colaboración fueron EE. UU. (194); Brasil (53); España (49); México (43); Francia (34); Italia (27); Argentina (26), y Canadá (26). Si bien la colaboración entre países está centrada en la colaboración entre Perú-EE. UU., existen varios países con los que se colabora de forma intensa e independiente de EE. UU, como Argentina, Austria, Chile, China y Reino Unido.

La institución peruana más productiva es el INEN con 121 artículos (37\% de los artículos peruanos), seguido por la Universidad Peruana Cayetano Heredia (UPCH) -77 artículos-. EI INEN desarrolló $88(72,7 \%)$ de sus artículos en colaboración, participando en total con 180 instituciones de 46 países, siendo el colaborador más frecuente la

Tabla 2. Publicaciones científicas y ensayos clínicos peruanos sobre cáncer publicados en revistas indizadas en SCOPUS o ISI, 2000-2011.

\begin{tabular}{|c|c|c|c|c|}
\hline & Total & Ensayos clínicos & Artículos originales * & Otros artículos ${ }^{\dagger}$ \\
\hline Variables & N. ${ }^{\circ}(\%)$ & N. ${ }^{\circ}(\%)$ & N. ${ }^{\circ}(\%)$ & N. ${ }^{\circ}(\%)$ \\
\hline \multicolumn{5}{|c|}{ Autor corresponsal } \\
\hline Extranjero & $251(70,1)$ & $45(86,5)$ & $143(70,1)$ & $63(61,8)$ \\
\hline Peruano & $107(29,9)$ & $7(13,5)$ & $61(29,9)$ & $39(38,2)$ \\
\hline \multicolumn{5}{|c|}{ Idioma de publicación } \\
\hline Inglés & $294(82,1)$ & $51(98,1)$ & $158(77,5)$ & $85(83,3)$ \\
\hline Otros & $64(17,9)$ & $1(1,9)$ & $46(22,6)$ & $17(16,7)$ \\
\hline \multicolumn{5}{|c|}{ Colaboración internacional } \\
\hline Sí & $246(68,7)$ & $46(88,5)$ & $134(65,7)$ & $66(64,7)$ \\
\hline No & $112(31,3)$ & $6(11,5)$ & $70(34,3)$ & $36(35,3)$ \\
\hline \multicolumn{5}{|c|}{ Revista de cáncer } \\
\hline Sí & $121(33,8)$ & $32(61,5)$ & $54(26,5)$ & $35(34,3)$ \\
\hline No & $237(66,2)$ & $20(38,5)$ & $150(73,5)$ & $67(65,7)$ \\
\hline \multicolumn{5}{|c|}{ Periodo de publicación } \\
\hline 2006-2011 & $261(72,9)$ & $46(88,5)$ & $133(65,2)$ & $82(80,4)$ \\
\hline $2000-2005$ & $97(27,1)$ & $6(11,5)$ & $71(34,8)$ & $20(19,6)$ \\
\hline \multicolumn{5}{|l|}{ Autoría INEN } \\
\hline Sí & $121(33,8)$ & $29(55,8)$ & $57(27,9)$ & $35(34,3)$ \\
\hline No & $237(66,2)$ & $23(44,2)$ & $147(72,1)$ & $67(65,7)$ \\
\hline \multicolumn{5}{|c|}{ Colaboración institucional } \\
\hline Sí & $269(75,1)$ & $46(88,5)$ & $152(74,5)$ & $71(69,6)$ \\
\hline No & $89(24,9)$ & $6(11,5)$ & $52(25,5)$ & $31(30,4)$ \\
\hline
\end{tabular}

*Artículos originales que no eran ensayos clínicos.

† Incluye reportes de caso, cartas al editor, revisiones, editoriales y otros. 
UPCH (Figura 2), con quien publicó ocho artículos. De otro lado, la UPCH realizó $62(80,5 \%)$ de sus artículos en colaboración, participando en total con 100 instituciones de 30 países, siendo su colaborador más frecuente la Washington University, con quien colaboró en 17 artículos. Además, colaboró de forma intensa (más de diez artículos) con nueve instituciones (Figura 2).

\section{DISCUSIÓN}

La producción científica sobre cáncer en el Perú se está incrementando más rápidamente que en otros temas de salud ${ }^{(8)}$, y con una mayor realización de ensayos clínicos. Esta situación es coherente con el mayor registro de ensayos clínicos sobre oncología en Perú (11). Además, se muestra que hay ensayos clínicos liderados por peruanos o con coautoría peruana, particularmente del INEN, aspecto que va contra la creencia de que los médicos que participan de ensayos clínicos en el Perú solo lo hacen como "recolectores de datos" (12).

El comportamiento de la investigación en cáncer en Perú es similar a lo que sucede a nivel mundial, con una mayor producción en cáncer de mama ${ }^{(13)}$, pero muy pobre en otros tipos de cáncer que generan alta mortalidad, como el cáncer de próstata, pulmón e hígado (Figura 2), situación parecida a Brasil, donde hay una pobre asociación entre la carga de enfermedad por cáncer y las investigaciones realizadas ${ }^{(14)}$.

EI INEN es la institución líder en investigación en cáncer con mayor número de publicaciones y colaboraciones; sin embargo, estas son de poca intensidad (es decir, publica colaborativamente con muchas instituciones, pero estas colaboraciones generan pocos artículos en común). Esto podría deberse a varios factores, como el que la intensidad de las colaboraciones depende del tiempo de trabajo en conjunto y el desarrollo de investigaciones clínicas en cáncer requiere largos tiempos de seguimiento. Es posible que la intensidad de las redes del INEN aumente en el futuro si se continúa con una política de colaboración con instituciones específicas.

La UPCH produce $40 \%$ menos artículos que el INEN en cáncer, y tiene menos colaboraciones; sin embargo, estas son más intensas. Esto podría deberse a que la UPCH es una institución que, probablemente, desarrolla trabajos en áreas específicas, como epidemiología, o en grupos de investigación ya conformados. Al respecto, estudios previos en diferentes disciplinas han mostrado que hay instituciones que tienen pocas colaboraciones pero con "vínculos fuertes" (15), vínculos que resultan fundamentales para asegurar la conectividad, acceso y difusión de la información; además, los vínculos de colaboración consolidados se han asociado con la continuidad y la estabilidad en la investigación, y con un mejor desempeño científico frente a las colaboraciones esporádicas ${ }^{(16)}$.

La RINC-UNASUR es una oportunidad para crear y fortalecer redes de investigación en cáncer a nivel regional, y los convenios que se vienen realizando para trabajo en conjunto verán reflejados sus efectos en futuras publicaciones. Esto se ha visto en la red de colaboración en cáncer denominada Arizon Cancer Coalition, cuyo análisis mostró que las políticas gubernamentales implementadas para unir diversos centros tuvieron como impacto la integración o formación de redes en investigación (17).

Las redes colaborativas más frecuentes guardan relación con instituciones estadounidenses; este comportamiento puede explicarse debido a la alta producción científica con filiación de EE. UU., líder en producción científica en cáncer ${ }^{(18,19)}$ en revistas de alto impacto en ISI y Medline.

Nuestro estudio tiene limitaciones. No incluimos bases de datos regionales como LILACS debido a nuestro interés en evaluar la producción de alta visibilidad e impacto reflejada en las bases elegidas, esto implica incluir aproximadamente $10 \%$ artículos menos que los realmente generados por instituciones peruanas. Por lo tanto, esta investigación no refleja la totalidad de la investigación peruana en cáncer. Sin embargo, esto no afectaría nuestro análisis de colaboración internacional, dado que representa menos del $7 \%$ de la producción en revistas peruanas ${ }^{(20)}$, y parte de ella ya ha sido recogida a través de las revistas indizadas en SCOPUS. Así mismo, incluir únicamente estas bases hace que nuestro estudio sea comparable a otras investigaciones sobre producción científica en cáncer ${ }^{(18,19)}$. Otra limitación se refiere a su restricción temporal al periodo 2000-2011; sin embargo, la producción anterior al año 2000 en revistas de alto impacto es esporádica. Finalmente, debemos mencionar que no hicimos la evaluación de la calidad de los artículos encontrados, debido a la diversidad de diseños y difícil homogenización, situación que podría requerir una futura investigación.

Aun así, este estudio muestra que la producción científica peruana en cáncer publicada en revistas de alto impacto se incrementa cada año, la institución más productiva y con mayor número de colaboraciones es el INEN, pero la UPCH tiene colaboraciones más intensas. Los ensayos clínicos representan un importante porcentaje de las investigaciones en cáncer. Se investiga más en cáncer de mama y cuello uterino, pero es necesario 
incrementar la producción en cáncer en general y, en particular, en aquellos de importante mortalidad en el Perú como el cáncer de cuello uterino, próstata, pulmón e hígado.

Contribuciones de autoría: PMT tuvo la idea y concepción del estudio, PMT, $\mathrm{CH}$ y JJMI recolectaron los datos, $\mathrm{PMT}, \mathrm{CH}$, SMF y GGA analizaron e interpretaron los datos, PMT y $\mathrm{CH}$ redactaron el manuscrito, JJMI, SMF y GGA hicieron la revisión crítica, todos los autores aprobaron la versión final del artículo.

Fuentes de financiamiento: autofinanciado.

Conflictos de interés: CSF es jefe del departamento de investigación del Instituto Nacional de Enfermedades Neoplásicas. Los demás autores declaran no tener conflictos de interés.

\section{REFERENCIAS BIBLIOGRÁFICAS}

1. Jemal A, Bray F, Center MM, Ferlay J, Ward E, Forman D. Global cancer statistics. CA Cancer J Clin. 2011;61(2):69-90.

2. Farmer P, Frenk J, Knaul FM, Shulman LN, Alleyne G, Armstrong L, et al. Expansion of cancer care and control in countries of low and middle income: a call to action. Lancet. 2010;376(9747):1186-93.

3. Cao Y, DePinho RA, Ernst M, Vousden K. Cancer research: past, present and future. Nat Rev Cancer. 2011;11(10):749-54.

4. Lewison G, Purushotham A, Mason M, McVie G, Sullivan R. Understanding the impact of public policy on cancer research: a bibliometric approach. Eur J Cancer. 2010;46(5):912-9.

5. Katz JS, Martin BR. What is research collaboration? Res Policy. 1997;26(1):1-18.

6. Ynalvez MA, Shrum WM. Professional networks, scientific collaboration, and publication productivity in resourceconstrained research institutions in a developing country. Res Policy. 2011;40(2):204-16.

7. Chinchilla-Rodríguez Z, BenaventPérez M, de Moya-Anegón F, Miguel S. International collaboration in medical research in Latin America and the Caribbean (2003-2007). J Am Soc Inf Sci Tech. 2012;11(63):2223-38.

8. Huamani C, Mayta-Tristan P. Producción científica peruana en medicina y redes de colaboración, análisis del Science Citation Index 2000-2009. Rev Peru Med Exp Salud Publica. 2010;27(3):315-25.

9. Suarez-Ognio L. Situación de Salud del Perú, riesgos y desafíos. Ponencia presentada en el Ciclo Internacional de Psicología Clínica. Universidad Peruana de Ciencias Aplicadas (UPC); 3 de mayo de 2012; Surco, Perú. Lima: UPC; 2012.

10. Kamada T, Kawai S. An algorithm for drawing general undirected graphs. Inform Process Lett. 1989;31:7-15.

11. Minaya G, Fuentes D, Obregon C, Ayala-Quintanilla B, Yagui M. Características de los ensayos clínicos autorizados en el Perú, 1995-2012. Rev Peru Med Exp Salud Publica. 2012;29(4):431-6.

12. Málaga G, Zuñiga-Rivera A. ¿Contribuyen los ensayos clínicos al desarrollo de la investigación en el Perú?̨cómo lograrlo?. Rev Peru Med Exp Salud Publica. 2012;29(4):529-34.

13. Glynn RW, Chin JZ, Kerin MJ Sweeney KJ. Representation of cancer in the medical literature -- a bibliometric analysis. PLoS One. 2010;5(11):e13902.

14. Saad ED, Pinheiro CM, Masson AL, Borghesi G, Hoff PM, Prisco FE. Increasing output and low publication rate of Brazilian studies presented at the American Society of Clinical Oncology Annual Meetings. Clinics. 2008;63(3):293-6

15. Abbasi A, Hossain L, Uddin S, Rasmussen K. Evolutionary dynamics of scientific collaboration networks: multi-levels and cross-time analysis. Scientometrics. 2011;89(2):687-710.

16. Gonzalez-Alcaide G, Park J, Huamani C, Gascón J, Ramos JM. Scientific authorships and collaboration network analysis on Chagas disease: papers indexed in PubMed (19402009). Rev Inst Med Trop S Paulo. 2012;54(4):219-28.

17. Provan KG, Leischow SJ, Keagy J, Nodora J. Research collaboration in the discovery, development, and delivery networks of a statewide cancer coalition. Eval Progr Plann. 2010;33(4):349-55.

18. Micheli A, Di Salvo F, Lombardo C, Ugolini D, Baili P, Pierotti M. Cancer research performance in the European Union: a study of published output from 2000 to 2008. Tumori. 2011;97(6):683-9.

19. Liu XY, Wan XH, Li ZW. Ten-year survey on oncology publications from China and other top-ranking countries. Chin Med J (Engl). 2011;124(20):3314-9.

20. Huamaní C, Pacheco-Romero J. Colaboración científica en artículos científicos de revistas biomédicas peruanas, 2005 - 2008. An Fac Med (Lima). 2011;72(4):261-8.

Correspondencia: Percy Mayta-Tristán

Dirección: Av. Brasil 2169, Dpto. 802, Lima 11, Perú.

Teléfono: (51) 987-532-133

Correoelectrónico:percy.mayta@upc.edu.pe 